\title{
Evasion and prediction II
}

\author{
JÖRG BRENDLE ${ }^{1}$ \\ Mathematisches Institut der Universität, Auf der Morgenstelle 10, 72076 Tübingen, \\ Germany; email: jobr@michelangelo.mathematik.uni-tuebingen.de \\ SAHARON ShElah ${ }^{2,3}$ \\ Department of Mathematics, Hebrew University, Givat Ram, 91904 Jerusalem, Israel \\ and \\ Department of Mathematics, Rutgers University, New Brunswick, NJ 08854, USA
}

\begin{abstract}
A subgroup $G \leq \mathbb{Z}^{\omega}$ exhibits the Specker phenomenon if every homomorphism $G \rightarrow \mathbb{Z}$ maps almost all unit vectors to 0 . We give several combinatorial characterizations of the cardinal $\mathfrak{s e}$, the size of the smallest $G \leq \mathbb{Z}^{\omega}$ exhibiting the Specker phenomenon. We also prove the consistency of $\mathfrak{b}<\mathfrak{e}$, where $\mathfrak{b}$ is the unbounding number and $\mathfrak{e}$ the evasion number. Our results answer several questions addressed by Blass.

1991 Mathematics subject classification. 03E05 03E35 20K20 20K25

Key words and phrases. Specker phenomenon, Cardinal invariants of the continuum, Forcing, Evading and predicting

1 Supported by DFG-grant Nr. Br 1420/1-1.

2 Supported by the Edmund Landau Center for research in Mathematical Analysis (sponsored by the MINERVA-foundation (Germany)).

3 publication number 540
\end{abstract}




\section{Introduction}

Specker [Sp] proved that given a homomorphism $h$ from $\mathbb{Z}^{\omega}$ to the infinite cyclic group $\mathbb{Z}$, where $\mathbb{Z}^{\omega}$ denotes the direct product of countably many copies of $\mathbb{Z}$, we have $h\left(e_{n}\right)=0$ for all but finitely many unit vectors $e_{n} \in \mathbb{Z}^{\omega}$ (in other words, the $n$-th component of $e_{n}$ is 1 , and its other components are 0). Blass [Bl] studied the Specker-Eda number $\mathfrak{s e}$, the size of the smallest subgroup $G \leq \mathbb{Z}^{\omega}$ containing all unit vectors which still has the property that every homomorphism $h: G \rightarrow \mathbb{Z}$ annihilates almost all unit vectors. We will give various (mostly less algebraic) characterizations of $\mathfrak{s e}$ (some of which already play a prominent role in Blass' work); we will also study some related cardinal invariants of the continuum.

To be more explicit, let $\leq *$ denote the eventual domination order on the Baire space $\omega^{\omega}$; i.e. $f \leq^{*} g$ iff $f(n) \leq g(n)$ for all but finitely many $n$. We shall usually abbreviate the statement in italics by $\forall^{\infty} n$; similarly we will write $\exists^{\infty} n$ for there are infinitely many $n$. The unbounding number $\mathfrak{b}$ is the smallest size of $a \leq^{*}-$ unbounded family $\mathcal{F}$ of functions in $\omega^{\omega}$ (i.e., given any $g \in \omega^{\omega}$, there is $f \in \mathcal{F}$ with $\left.\exists^{\infty} n(f(n)>g(n))\right)$. Given a $\sigma$-ideal $\mathcal{I}$ on $\omega^{\omega}$, the additivity $\operatorname{add}(\mathcal{I})$ is the least cardinality of a family $\mathcal{F}$ of members of $\mathcal{I}$ whose union is not in $\mathcal{I}$. We shall use this cardinal only in the cases $\mathcal{I}=\mathcal{M}$, the ideal of meager sets, and $\mathcal{I}=\mathcal{L}$, the ideal of Lebesgue null sets. - While the preceding invariants have been studied by a number of people in the last two decades, the following concept was introduced only recently by Blass [Bl]. Given an at most countable set $S$, an $S$-valued predictor is a pair $\pi=\left(D_{\pi},\left\langle\pi_{n} ; n \in D_{\pi}\right\rangle\right)$ where $D_{\pi} \subseteq \omega$ is infinite and for each $n \in D_{\pi}$, $\pi_{n}$ is a function from $S^{n}$ to $S$. $\pi$ predicts $f \in S^{\omega}$ iff for all but finitely many $n \in D_{\pi}$, we have $f(n)=\pi_{n}(f\lceil n)$; otherwise $f$ evades $\pi$. The evasion number $\mathfrak{e}$ is the smallest size of a family $\mathcal{F}$ of functions in $\omega^{\omega}$ such that no $\omega$-valued predictor predicts all $f \in \mathcal{F}$. A $\mathbb{Z}$-valued predictor is linear iff all $\pi_{n}: \mathbb{Z}^{n} \rightarrow \mathbb{Q}$ are $\mathbb{Q}$-linear maps. The corresponding linear evasion number shall be denoted by $\mathfrak{e}_{\ell}$ (i.e., $\mathfrak{e}_{\ell}=\min \left\{|\mathcal{F}| ; \mathcal{F} \subseteq \mathbb{Z}^{\omega}\right.$ and no linear $\mathbb{Z}$-valued predictor predicts all $f \in \mathcal{F}\}$ ). (Blass' definition of linear evading [Bl, section 4] is slightly different; however, it gives rise to the same cardinal; we use the present definition because we shall work with functions in $\mathbb{Z}^{\omega}$ in 2.2.)

These notions enable us to phrase our main results. 
Theorem A. It is consistent with $Z F C$ to assume $\mathfrak{b}<\mathfrak{e}$.

Theorem B. $\mathfrak{s e}=\mathfrak{e}_{\ell}=\min \{\mathfrak{e}, \mathfrak{b}\}$.

They will be proved in sections 1 and 2 of our work. Section 2 also contains a further purely combinatorial characterization of the cardinal $\mathfrak{s e}$ (subsections 2.4 and 2.5). To put our results into a somewhat larger context, we point out the following consequences which involve some earlier results, due mostly to Blass [Bl].

Corollary. (a) $\operatorname{add}(\mathcal{L}) \leq \mathfrak{s e} \leq \operatorname{add}(\mathcal{M}) \leq \mathfrak{b}$;

(b) any of the inequalities in (a) can be consistently strict;

(c) it is consistent with $Z F C$ to assume $\mathfrak{e}_{\ell}<\mathfrak{e}$.

Theorems A and B together with the Corollary give a complete solution to Questions (1) through (3) in [Bl, section 5]. Note in particular that the cardinals (2) through (5) in Corollary 8 in [Bl, section 3$]$ are indeed equal.

Proof of Corollary. (a) This follows from Theorem B and Blass' results [Bl, Theorems 12 and 13]. The well-known inequality $\operatorname{add}(\mathcal{M}) \leq \mathfrak{b}$ is due to Miller [Mi].

(b) The consistency of $\operatorname{add}(\mathcal{M})<\mathfrak{b}$ is well-known (it holds e.g. in the Mathias or Laver real models); for the consistency of $\operatorname{add}(\mathcal{L})<\mathfrak{s e}$ see $[\mathrm{Bl}]$ (in particular [Bl, Theorem 9]); the consistency of $\mathfrak{s e}<\operatorname{add}(\mathcal{M})$ follows from Theorem B and [Br, Theorem A].

(c) This is immediate from Theorems A and B.

A set of reals predicted by a single predictor is small in various senses; it belongs, in particular, both to $\mathcal{M}$ and $\mathcal{L}$. This motivates us to introduce the $\sigma$-ideal $\mathcal{J}$ on $\omega^{\omega}$ generated by such sets of reals (see [Br, section 4] for more on this). Clearly, the uniformity of $\mathcal{J}$ (i.e., the size of the smallest set of reals not in $\mathcal{J}$ ) is closely related to the evasion number. In fact, $\mathfrak{e} \leq \mathfrak{e}(\omega)$ where $\mathfrak{e}(\omega)$ denotes the former cardinal. We shall show in section 3 that these two cardinals are equal under some additional assumption, thus giving a partial answer to [Br, section 6, question (4)].

The results of this work are due to the second author. It was the first author's task to work them out and to write up the paper.

Notational remarks. A p.o. $\mathbb{P}$ is $\sigma$-centered iff there are $\mathbb{P}_{n} \subseteq \mathbb{P}(n \in \omega)$ so that $\mathbb{P}=\bigcup_{n} \mathbb{P}_{n}$ and given $n \in \omega, F \subseteq \mathbb{P}_{n}$ finite, there is $p \in \mathbb{P}$ extending all $q \in F$. $\mathbb{P}$-names are denoted by symbols like $\dot{h}, \dot{\pi}, \dot{D}, \ldots \mid$ stands for divides; $\chi$ means does not divide. 
Acknowledgment. The first author thanks the Wrocław logic group for several discussions about the material presented here.

\section{$\S 1$. Proof of Theorem A}

1.1. We shall use a finite support iteration of $c c c$ p.o.'s of length $\kappa$ (where $\kappa \geq \omega_{2}$ is a regular cardinal) over a model $V$ for $C H$ to prove the consistency of $\mathfrak{e}>\mathfrak{b}$. In fact, in the resulting model, $\mathfrak{b}=\omega_{1}$ and $\mathfrak{e}=\kappa$. We start with defining the p.o. $\mathbb{P}$ we want to iterate. Notice that it is quite similar to the one used in [Br, 4.3.] for predicting below a given function.

$$
\begin{aligned}
\langle d, \pi, F\rangle \in \mathbb{P} \Longleftrightarrow d \in 2^{<\omega} \text { is a finite partial function, } \\
\qquad \begin{aligned}
\pi & =\left\langle\pi_{n} ; n \in d^{-1}(\{1\})\right\rangle \text { and } \pi_{n}: \omega^{n} \rightarrow \omega \text { is a finite partial function, } \\
& F \subseteq \omega^{\omega} \text { is finite and }(f \neq g \in F \longrightarrow \max \{n ; f\lceil n=g\lceil n\}<|d|) .
\end{aligned}
\end{aligned}
$$

The order is given by:

$$
\begin{aligned}
\left\langle d^{\prime}, \pi^{\prime}, F^{\prime}\right\rangle \leq\langle d, \pi, F\rangle \Longleftrightarrow & d^{\prime} \supseteq d, \pi^{\prime} \supseteq \pi, F^{\prime} \supseteq F \text { and } \\
& \left(f \in F, n \in\left(d^{\prime}\right)^{-1}(\{1\}) \backslash d^{-1}(\{1\}) \longrightarrow \pi_{n}^{\prime}(f\lceil n)=f(n))\right.
\end{aligned}
$$

(in particular $\pi_{n}^{\prime}(f\lceil n)$ is defined).

Notice that we use the convention that stronger conditions are smaller in the p.o. - The first two coordinates of a condition are intended as a finite approximation to a generic predictor; the third coordinate then guarantees that functions are predicted from some point on. Thus it is straightforward that $\mathbb{P}$ adjoins a predictor which predicts all groundmodel functions. Hence iterating $\mathbb{P}$ increases $\mathfrak{e}$.

Furthermore $\mathbb{P}$ is $\sigma$-centered (and thus in particular $c c c$ ). To see this simply notice that conditions with the same initial segment in the first two coordinates are compatible.

So it remains to show that $\mathfrak{b}=\omega_{1}$ after iterating $\mathbb{P}$. For this it suffices to show the following:

(*) whenever $G \in W$ is an unbounded family of functions from $\omega$ to $\omega$, and $\mathbb{P} \in W$ is the p.o. defined above, then

$$
\|_{\mathbb{P}} \text { " } G \text { is unbounded". }
$$


Using $(*)$ we can show that $\omega^{\omega} \cap V$ is still unbounded in the final model: $(*)$ guarantees that it stays unbounded in successor steps of the iteration; and one of the usual preservation results for finite support iterations (see, e.g., [JS, Theorem 2.2]) shows that it does so in limit steps of the iteration as well. Now, $V \models C H$; hence $\omega^{\omega} \cap V$ is an unbounded family of size $\omega_{1}$ in the final model.

To start with the proof of $(*)$, let $\dot{h}$ be a $\mathbb{P}$-name for a function in $\omega^{\omega}$. For each $d \in 2^{<\omega}, \pi=\left\langle\pi_{n} ; n \in d^{-1}(\{1\})\right\rangle$ an initial segment of a predictor (as in the definition of $\mathbb{P}), k \in \omega$ and $\bar{f}^{*}=\left\langle f_{\ell}^{*} \in \omega^{|d|} ; \ell<k\right\rangle$ we define $h=h_{d, \pi, \bar{f}^{*}} \in(\omega+1)^{\omega}$ by

$$
\begin{gathered}
h(n):=\min \left\{m \leq \omega ; \text { for no } p \in \mathbb{P} \text { with } p=\langle d, \pi, F\rangle, F=\left\{f_{\ell} ; \ell<k\right\}, f_{\ell}|| d \mid=f_{\ell}^{*},\right. \\
\text { do we have } \left.p \models_{\mathbb{P}} \text { “ } \dot{h}(n)>m \text { " }\right\} .
\end{gathered}
$$

\subsection{Main Claim. $h \in \omega^{\omega}$.}

1.3. Proof of $(*)$ From the Main Claim. Let $h^{*} \in \omega^{\omega}$ such that for all $d, \pi, \bar{f}^{*}$ as above we have $h_{d, \pi, \bar{f}^{*}} \leq^{*} h^{*}$. As $G$ is unbounded we can find $f \in G$ such that there are infinitely many $n$ with $f(n)>h^{*}(n)$. We claim that $\|-_{\mathbb{P}} " \exists \exists^{\infty} n(f(n)>\dot{h}(n))$ ". This will show $(*)$.

Assume $m \in \omega$ and $p \in \mathbb{P}$ are such that

$$
p \models_{\mathbb{P}} \text { " } \forall n \geq m(f(n) \leq \dot{h}(n)) " .
$$

Find $d, \pi, \bar{f}^{*}$ such that $p=\langle d, \pi, F\rangle$ where $F=\left\{f_{\ell} ; \ell<k\right\}$ and $f_{\ell}|| d \mid=f_{\ell}^{*}$. Find $n \geq m$ such that $f(n)>h^{*}(n)$ and $h^{*}(n) \geq h_{d, \pi, \bar{f}^{*}}(n)$. Then

$$
p \models_{\mathbb{P}} " h_{d, \pi, \bar{f}^{*}}(n)<f(n) \leq \dot{h}(n) ",
$$

contradicting the definition of $h_{d, \pi, \bar{f}^{*}}$.

1.4. Proof of the Main Claim (1.2.). Let $d, \pi, k, \bar{f}^{*}=\left\langle f_{\ell}^{*} ; \ell<k\right\rangle$ as above and $n \in \omega$ be fixed. Now assume that we have $p_{i}=\left\langle d, \pi,\left\{f_{\ell}^{i} ; \ell<k\right\}\right\rangle$ with $f_{\ell}^{i}|| d \mid=f_{\ell}^{*}$ and

$$
p_{i} \models_{\mathbb{P}} " \dot{h}(n)>i " .
$$

We shall reach a contradiction. As we can replace $\left\langle p_{i} ; i \in \omega\right\rangle$ by a subsequence, if necessary, we may assume that for all $\ell<k$ :

either $(a)_{\ell}$ for some $g_{\ell} \in \omega^{\omega}$ for all $i\left(f_{\ell}^{i}\left\lceil i=g_{\ell}\lceil i)\right.\right.$

or $(b)_{\ell}$ for some $i_{\ell} \in \omega$ and $\hat{g}_{\ell} \in \omega^{i_{\ell}}\left(f_{\ell}^{i}\left\lceil i_{\ell}=\hat{g}_{\ell} \wedge f_{\ell}^{i}\left(i_{\ell}\right)>i\right)\right.$. 


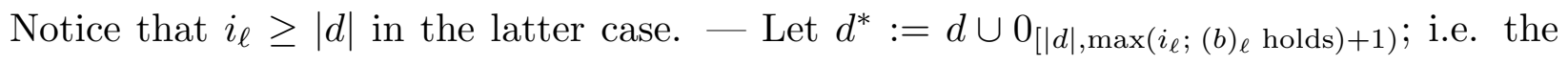
function $d^{*}$ takes value 0 between $|d|$ and the maximum of the $i_{\ell}$. Put $F^{*}:=\left\{g_{\ell} ;(a)_{\ell}\right.$ holds $\}$. Then clearly $p^{*}=\left\langle d^{*}, \pi, F^{*}\right\rangle \in \mathbb{P}$. Now choose $\ell^{*}$ and $q \leq p^{*}$ such that

$$
q \Vdash_{\mathbb{P}} " \dot{h}(n)=\ell^{*} " .
$$

We shall find $i>\ell^{*}$ so that $q$ and $p_{i}$ are compatible; this is a contradiction because $q$ and $p_{i}$ force contradictory statements.

Assume $q=\left\langle d^{q}, \pi^{q}, F^{q}\right\rangle$. Choose $i \geq \ell^{*}$ large enough such that:

(A) $i \geq\left|d^{q}\right|$;

(B) $i \geq \max \left\{\max \left\{\sigma(j) ; \sigma \in \operatorname{dom}\left(\pi_{m}^{q}\right) \wedge j \in m\right\} ; m \in\left(d^{q}\right)^{-1}(\{1\})\right\}$.

Notice that $(\mathrm{A})$ implies that $\left.f_{\ell}^{i}\right\rceil\left|d^{q}\right|=g_{\ell}|| d^{q} \mid$ whenever $(a)_{\ell}$ holds, while $f_{\ell}^{i}\left(i_{\ell}\right)>\max \{\max$ $\left.\left\{\sigma(j) ; \sigma \in \operatorname{dom}\left(\pi_{m}^{q}\right) \wedge j \in m\right\} ; m \in\left(d^{q}\right)^{-1}(\{1\})\right\}$ by (B) in case $(b)_{\ell}$ holds. For such $i$ let $q^{i}=\left\langle d^{i}, \pi^{i}, F^{i}\right\rangle$ where

- $d^{i}=d^{q} \cup 0_{\left[\left|d^{q}\right|, a\right)}$, where $a$ is large enough such that all functions in $F^{i}$ disagree before $a$;

- $\pi^{i} \supseteq \pi^{q}$ such that for all $m \in\left(d^{q}\right)^{-1}(\{1\}) \backslash d^{-1}(\{1\})$ and all $f_{\ell}^{i}$ so that $(b)_{\ell}$ holds, we have

$$
f_{\ell}^{i}(m)=\pi_{m}^{i}\left(f_{\ell}^{i}\lceil m)\right.
$$

(This can be done because, by (B), $\pi_{m}^{q}$ was not defined yet for sequences of the form $f_{\ell}^{i}\lceil m$.)

$-F^{i}=F^{q} \cup\left\{f_{\ell}^{i} ; \ell<k\right\}$.

Now we clearly have $q^{i} \in \mathbb{P}$ and $q^{i} \leq q$. So we are left with checking $q^{i} \leq p_{i}$. The inclusion relations are all satisfied. Hence it suffices to see that for all $\ell<k$ and $m \in$ $\left(d^{i}\right)^{-1}(\{1\}) \backslash d^{-1}(\{1\})$, we have

$$
f_{\ell}^{i}(m)=\pi_{m}^{i}\left(f_{\ell}^{i}\lceil m)\right.
$$

In case $(b)_{\ell}$ holds this is true by $(\star)$. In case $(a)_{\ell}$ holds we have $f_{\ell}^{i}\left\lceil(m+1)=g_{\ell}\lceil(m+1)\right.$ for all such $m$. As $q \leq p^{*}$ we have $\pi_{m}^{i}\left(g_{\ell}\lceil m)=\pi_{m}^{q}\left(g_{\ell}\lceil m)=g_{\ell}(m)\right.\right.$ for such $m$, and (+) holds again. This completes the proof of the Main Claim. 


\section{§2. Proof of Theorem B}

\subsection{Theorem. $\mathfrak{s e} \leq \mathfrak{e}$.}

Proof. Let $\mathcal{F} \subseteq \omega^{\omega},|\mathcal{F}|<\mathfrak{s e}$. By Blass' result $\mathfrak{s e} \leq \mathfrak{b}\left[\mathrm{Bl}\right.$, Theorem 2], there is $g \in \omega^{\omega}$ such that for all $f \in \mathcal{F} \forall^{\infty} n(f(n)<g(n))$. Without loss $g$ is strictly increasing. We let $\left\langle p_{n} ; n \in \omega\right\rangle$ be a sequence of distinct primes such that $p_{n}>>g(n)$ and $p_{n}>>\prod_{\ell<n} p_{\ell}$. For $f \in \mathcal{F}$, let $a_{f} \in \omega^{\omega}$ be defined by

$$
a_{f}(n):=f(n) \cdot \prod_{\ell \leq n} p_{\ell}
$$

Let $G \leq \mathbb{Z}^{\omega}$ be the pure closure of the subgroup generated by the unit vectors $e_{n}, n \in \omega$, and the $a_{f}, f \in \mathcal{F}$. Clearly $|G|<\mathfrak{s e}$. Hence there is $h: G \longrightarrow \mathbb{Z}$ a homomorphism such that $W:=\left\{n ; h\left(e_{n}\right) \neq 0\right\}$ is infinite.

Let us define

$$
W^{*}:=\left\{n \in \omega ; \exists i>n\left(p_{i} \mid h\left(e_{m}\right) \text { whenever } m \in\{n+1, \ldots, i-1\} \text { but } p_{i} \not h\left(e_{n}\right)\right)\right\} .
$$

We claim that $W^{*}$ is an infinite subset of $W$. To see this, first note that trivially $W^{*} \subseteq W$, by the clause $p_{i} \backslash h\left(e_{n}\right)$. Next, given $n_{0} \in W$, find $i>n_{0}$ so that $p_{i} \backslash h\left(e_{n_{0}}\right)$. Then clearly there is $n \geq n_{0}$ so that $n \in W$ and $p_{i} \backslash h\left(e_{n}\right)$ and for all $m \in\{n+1, \ldots, i-1\}, p_{i} \mid h\left(e_{m}\right)$. Thus $n \in W^{*}$. This shows that $W^{*}$ is infinite.

We introduce a predictor $\pi=\left(W^{*},\left\langle\pi_{n} ; n \in W^{*}\right\rangle\right)$ as follows. Given $n \in W^{*}$ and $s \in \omega^{n}$ so that max $r n g(s)<g(n-1)$, if there is $f \in \mathcal{F}$ with $s \subseteq f$ and $f(n)<g(n)$ and $\left|h\left(a_{f}\right)\right|<p_{n-1}$, then let $\pi_{n}(s)=f(n)$ for some $f$ with the above property. Otherwise $\pi_{n}(s)$ is arbitrary.

We claim that $\pi$ predicts all $f \in \mathcal{F}$. This clearly finishes the proof. Assume this were false, i.e. there is $f \in \mathcal{F}$ which evades $\pi$. Let $n \in W^{*}$ be large enough, such that $\max r n g\left(f\lceil n)<g(n-1), f(n)<g(n),\left|h\left(a_{f}\right)\right|<p_{n-1}\right.$ and $\pi_{n}(f\lceil n) \neq f(n)$. Then, by the definition of $\pi$, there must be $f^{\prime} \in \mathcal{F}$ with $f^{\prime}\left\lceil n=f\left\lceil n, f^{\prime}(n)<g(n),\left|h\left(a_{f^{\prime}}\right)\right|<p_{n-1}\right.\right.$ and $\pi_{n}\left(f^{\prime}\lceil n)=f^{\prime}(n) \neq f(n)\right.$. Now, for $k \in\left\{f, f^{\prime}\right\}$, we let

$$
\begin{aligned}
& a_{k}^{0}=\left(a_{k}(0), \ldots, a_{k}(n-1), 0, \ldots\right) \\
& a_{k}^{1}=\left(0, \ldots, 0, a_{k}(n), 0, \ldots\right) \\
& a_{k}^{2}=\left(0, \ldots, 0, a_{k}(n+1), \ldots, a_{k}(i-1), 0, \ldots\right) \\
& a_{k}^{3}=\left(0, \ldots, 0, a_{k}(i), a_{k}(i+1), \ldots\right)
\end{aligned}
$$


where $i$ witnesses that $n \in W^{*}$. So we have $a_{k}=a_{k}^{0}+a_{k}^{1}+a_{k}^{2}+a_{k}^{3}$. Thus

$$
h\left(a_{f^{\prime}}-a_{f}\right)=h\left(a_{f^{\prime}}^{0}-a_{f}^{0}\right)+h\left(a_{f^{\prime}}^{1}-a_{f}^{1}\right)+h\left(a_{f^{\prime}}^{2}-a_{f}^{2}\right)+h\left(a_{f^{\prime}}^{3}-a_{f}^{3}\right) .
$$

Clearly $h\left(a_{f^{\prime}}^{0}-a_{f}^{0}\right)=h(0)=0$. Next, $p_{i} \cdot \prod_{\ell \leq n} p_{\ell} \operatorname{divides} h\left(a_{f^{\prime}}^{3}-a_{f}^{3}\right)$ by definition of the $a_{k}$; it also divides $h\left(a_{f^{\prime}}^{2}-a_{f}^{2}\right)$ by definition of the $a_{k}$ and because $p_{i} \mid h\left(e_{m}\right)$ for $m \in\{n+1, \ldots, i-1\}$ as $i$ witnesses $n \in W^{*}$. Thus ( $\star$ ) yields the equation

$$
h\left(a_{f^{\prime}}-a_{f}\right)=h\left(a_{f^{\prime}}^{1}-a_{f}^{1}\right) \quad \text { in } \quad \mathbb{Z} /\left(p_{i} \cdot \prod_{\ell \leq n} p_{\ell}\right) \mathbb{Z} . \quad(\star \star)
$$

The right-hand side in ( $\star \star)$ must be non-zero, because $p_{i} \not h\left(e_{n}\right)$ (as $i$ witnesses $n \in W^{*}$ ) and $p_{i} \chi\left(a_{f^{\prime}}(n)-a_{f}(n)\right)=\prod_{\ell \leq n} p_{\ell} \cdot\left(f^{\prime}(n)-f(n)\right)\left(\right.$ as $\left.f^{\prime}(n), f(n)<g(n)<<p_{n}<<p_{i}\right)$. However, it certainly is divisible by $\prod_{\ell \leq n} p_{n}$, whereas the left-hand side in $(\star \star)$ is not unless it is zero (as $\left|h\left(a_{f}\right)\right|,\left|h\left(a_{f^{\prime}}\right)\right|<p_{n-1}<<p_{n}$ ). This shows that the equation $(\star \star)$ cannot hold, the final contradiction.

Note that this result improves [Br, Theorem 3.2].

2.2. Lemma. $\mathfrak{e}_{\ell} \geq \min \{\mathfrak{e}, \mathfrak{b}\}$.

Proof. Let $\mathcal{F} \subseteq \mathbb{Z}^{\omega},|\mathcal{F}|<\min \{\mathfrak{e}, \mathfrak{b}\}$. Find $g \in \omega^{\omega}$ strictly increasing so that for all $f \in \mathcal{F}$, we have $|f|<^{*} g$, where $|f|(n)=|f(n)|$. We partition $\omega$ into intervals $I_{n}, n \in \omega$, so that $\max \left(I_{n}\right)+1=\min \left(I_{n+1}\right)$, as follows. $I_{0}=\{0\}$. Assume $I_{n}$ is defined; choose $I_{n+1}$ so that $\left|I_{n+1}\right|>\left[2 \cdot g\left(\max \left(I_{n}\right)\right)\right]^{\sum_{i \leq n}\left|I_{i}\right|}$. For $f \in \mathcal{F}$, define $\bar{f}$ by $\bar{f}(n):=f\left\lceil I_{n}\right.$, and let $\overline{\mathcal{F}}=\{\bar{f} ; f \in \mathcal{F}\}$. Use $|\overline{\mathcal{F}}|<\mathfrak{e}$ to get a single predictor $\bar{\pi}=\left(\bar{D},\left\langle\bar{\pi}_{n} ; n \in \bar{D}\right\rangle\right)$ predicting all the $\bar{f} \in \overline{\mathcal{F}}$. For $n \in \bar{D}$, let $\Gamma_{n}:=r n g\left(\bar{\pi}_{n} \uparrow\left(-g\left(\max \left(I_{n-1}\right)\right), g\left(\max \left(I_{n-1}\right)\right)\right) \bigcup_{i<n} I_{i}\right) \cap \mathbb{Z}^{I_{n}}$. So $\left|\Gamma_{n}\right|<\left|I_{n}\right|$; hence for some $i_{n} \in I_{n}$, the vector $\bar{x}_{i_{n}}=\left\langle t\left(i_{n}\right) ; t \in \Gamma_{n}\right\rangle$ depends on the vectors $\left\{\bar{x}_{i}=\left\langle t(i) ; t \in \Gamma_{n}\right\rangle ; \min \left(I_{n}\right) \leq i<i_{n}\right\}$. Say $\bar{x}_{i_{n}}=\sum_{\min \left(I_{n}\right) \leq i<i_{n}} q_{i}^{n} \bar{x}_{i}$, where $q_{i}^{n} \in \mathbb{Q}$. In particular, for fixed $t \in \Gamma_{n}$, we have $t\left(i_{n}\right)=\sum_{\min \left(I_{n}\right) \leq i<i_{n}} q_{i}^{n} t(i)$. This allows us to define a linear predictor $\pi=\left(D,\left\langle\pi_{n} ; n \in D\right\rangle\right)$ with $D=\left\{i_{n} ; n \in \omega\right\}$ and $\pi_{i_{n}}(s)=$ $\sum_{\min \left(I_{n}\right) \leq i<i_{n}} q_{i}^{n} s(i)$. Note that if $n \in \omega$ is such that $\max r n g\left(|f| \uparrow \cup_{i<n} I_{i}\right)<g\left(\max \left(I_{n-1}\right)\right)$ and $\bar{\pi}_{n}\left(\bar{f}\lceil n)=\bar{f}(n)\right.$, then $\pi_{i_{n}}\left(f\left\lceil i_{n}\right)=f\left(i_{n}\right)\right.$. Hence, as $\bar{\pi}$ predicts all $\bar{f} \in \overline{\mathcal{F}}$, $\pi$ predicts all $f \in \mathcal{F}$.

2.3. Clearly, Theorem B follows from 2.1., 2.2. and Blass' results $\mathfrak{e}_{\ell} \leq \mathfrak{s e} \leq \mathfrak{b}[\mathrm{Bl}$, Theorem 2, Corollary 8 and Theorem 10]. 
2.4. Definition. Given $D \subseteq \omega$ infinite and $\bar{a}=\left\langle a_{n} \in[\omega] \leq n ; n \in D\right\rangle$, the slalom $S_{D}^{\bar{a}}$ is the set of all functions $f$ in $\omega^{\omega}$ with $f(n) \in a_{n}$ for almost all $n \in D$.

Using this notion we can give a combinatorial characterization of the cardinal $\mathfrak{e}_{\ell}=\mathfrak{s e}$.

2.5. Lemma. $\min \{\mathfrak{e}, \mathfrak{b}\}=\min \left\{|\mathcal{F}| ; \mathcal{F} \subseteq \omega^{\omega}\right.$ and for all $D \subseteq \omega$ and $\bar{a}=\left\langle a_{n} \in\right.$ $[\omega] \leq n ; n \in D\rangle$ there is $\left.f \in \mathcal{F} \backslash S_{D}^{\bar{a}}\right\}$.

Note. It is immediate that the cardinal on the right-hand side is bigger than or equal to the additivity of Lebesgue measure $\operatorname{add}(\mathcal{L})$, by Bartoszyński's characterization of that cardinal $([\mathrm{Ba} 1],[\mathrm{Ba} 2])$. We also note that the original proof of $\operatorname{add}(\mathcal{L}) \leq \operatorname{add}(\mathcal{M})[\mathrm{Ba} 1]$ shows in fact that this cardinal is $\leq \operatorname{add}(\mathcal{M})$ as well. This gives an alternative proof of Blass' $\min \{\mathfrak{e}, \mathfrak{b}\} \leq \operatorname{add}(\mathcal{M})[\mathrm{Bl}$, Theorem 13$]$.

Proof. " $\geq "$. By Theorem B, it suffices to show that $\mathfrak{e}_{\ell}$ is bigger than or equal to the cardinal on the right-hand side. However, this is exactly like Blass' original proof of $\operatorname{add}(\mathcal{L}) \leq \mathfrak{e}_{\ell}[\mathrm{Bl}$, Theorem 12], and we therefore leave details to the reader.

$" \leq "$. This argument is very similar to the one in Lemma 2.2. So we just stress the differences.

Take $\mathcal{F} \subseteq \omega^{\omega},|\mathcal{F}|<\min \{\mathfrak{e}, \mathfrak{b}\}$. Find $g$ strictly increasing and eventually dominating all functions from $\mathcal{F}$. As before, partition $\omega$ into intervals $I_{n}, n \in \omega$; this time we require that $i_{n+1}:=g\left(\max \left(I_{n}\right)\right)^{\sum_{i \leq n}\left|I_{i}\right|} \in I_{n+1} \cdot \bar{f}, \overline{\mathcal{F}}$ and $\bar{\pi}, \bar{D}$ are defined as before.

We put $D:=\left\{i_{n} ; n \in \bar{D}\right\}$ and $a_{i_{n}}=\left\{\bar{\pi}_{n}(s)\left(i_{n}\right) ; s \in g\left(\max \left(I_{n-1}\right)\right) \bigcup_{i<n} I_{i}\right\} \in[\omega] \leq i_{n}$, and leave it to the reader to check that $\mathcal{F} \subseteq S_{D}^{\bar{a}}$.

2.6. The notion of linear predicting can be generalized as follows (see [Br, section 4] for details). Let $\mathbb{K}$ be an at most countable field. A $\mathbb{K}$-valued predictor $\pi=\left(D_{\pi},\left\langle\pi_{n} ; n \in\right.\right.$ $\left.\left.D_{\pi}\right\rangle\right)$ is linear iff all $\pi_{n}: \mathbb{K}^{n} \rightarrow \mathbb{K}$ are linear. $\mathfrak{e}_{\mathbb{K}}$ is the corresponding linear evasion number. We easily see $\mathfrak{e}_{\mathbb{Q}}=\mathfrak{e}_{\ell}$. Rewriting the proof of 2.2. in this more general context gives $\mathfrak{e}_{\mathbb{K}} \geq \min \{\mathfrak{e}, \mathfrak{b}\}$ for arbitrary $\mathbb{K}$ and $\mathfrak{e}_{\mathbb{K}} \geq \mathfrak{e}$ in case $\mathbb{K}$ is finite. As $\mathfrak{e}_{\mathbb{K}} \leq \mathfrak{b}$ for infinite $\mathbb{K}[\mathrm{Br}, 5.4$.$] , we get \mathfrak{e}_{\mathbb{K}}=\min \{\mathfrak{e}, \mathfrak{b}\}$ for such fields - in particular all $\mathfrak{e}_{\mathbb{K}}$ for $\mathbb{K}$ a countable field are equal. We do not know whether this is true for finite $\mathbb{K}$. Note that $\mathfrak{e}_{\mathbb{K}}>\mathfrak{e}, \mathfrak{b}$ is consistent for such fields [Br, section 4]. 


\section{$\S 3$. Some results on evasion ideals}

3.1. Definition. We say a predictor $\pi=\left(D,\left\langle\pi_{n} ; n \in D\right\rangle\right)$ predicts a function $f \in \omega^{\omega}$ everywhere if $\pi_{n}(f \mid n)=f(n)$ holds for all $n \in D$. We put $\mathfrak{e}(\omega):=\min \left\{|\mathcal{F}| ; \mathcal{F} \subseteq \omega^{\omega} \wedge\right.$ for all countable families of predictors $\Pi$ there is $f \in \mathcal{F}$ evading all $\pi \in \Pi\}$, the uniformity of the evasion ideal $\mathcal{J}$. - As usual, $\operatorname{cov}(\mathcal{M})$ denotes the covering number of the ideal $\mathcal{M}$, i.e. the smallest size of a family $\mathcal{F} \subseteq \mathcal{M}$ so that $\bigcup \mathcal{F}=\omega^{\omega}$.

3.2. Observation. Assume $\left\langle D^{n} ; n \in \omega\right\rangle$ is a decreasing sequence of infinite subsets of $\omega$, and $\left\langle\pi^{n}=\left(D^{n},\left\langle\pi_{k}^{n} ; k \in D^{n}\right\rangle\right) ; n \in \omega\right\rangle$ is a sequence of predictors. Then there are a set $D \subseteq \omega$, almost included in all $D^{n}$, and a predictor $\pi=\left(D,\left\langle\pi_{k} ; k \in D\right\rangle\right)$ predicting all functions which are predicted by one of the $\pi^{n}$.

Proof. We can assume that each function which is predicted by some $\pi^{n}$ is predicted everywhere by some $\pi^{m}$ - otherwise go over to sequences $\left\langle E^{n} ; n \in \omega\right\rangle$ and $\left\langle\bar{\pi}^{n}=\left(E^{n},\left\langle\bar{\pi}_{k}^{n} ; k \in E^{n}\right\rangle\right) ; n \in \omega\right\rangle$ such that (i) for all $n \in \omega$ there is $m \in \omega$ so that $E^{m} \subseteq D^{n}$ and $\bar{\pi}_{k}^{m}=\pi_{k}^{n}$ for $k \in E^{m}$ and (ii) for all $n, m \in \omega$ there is $\ell \in \omega$ so that $E^{\ell} \subseteq E^{n} \backslash m$ and $\bar{\pi}_{k}^{\ell}=\bar{\pi}_{k}^{n}$ for $k \in E^{\ell}$.

Choose $d^{n} \in D^{n}$ minimal with $d^{n}>d^{n-1}$, and put $D=\left\{d^{n} ; n \in \omega\right\}$. Fix $n \in \omega$ and $s \in \omega^{d^{n}}$. To define $\pi_{d^{n}}(s)$, choose, if possible, $i \leq n$ minimal so that for all $k \in D^{i} \cap d^{n}$, we have $\pi_{k}^{i}(s \mid k)=s(k)$, and let $\pi_{d^{n}}(s)=\pi_{d^{n}}^{i}(s)$. If this is impossible, let $\pi_{d^{n}}(s)$ be arbitrary.

To see that this works, take $f \in \omega^{\omega}$ and $i \in \omega$ minimal so that $\pi^{i}$ predicts $f$ everywhere. As the set of functions predicted everywhere by a single predictor is closed, there are $n \geq i$ and $s \in \omega^{d^{n}}$ so that $s \subseteq f$ and $s$ is not predicted everywhere by any of the $\pi^{j}$ where $j<i$. Then $\pi_{d^{m}}\left(f\left\lceil d^{m}\right)=\pi_{d^{m}}^{i}\left(f\left\lceil d^{m}\right)\right.\right.$ for all $m \geq n$, as required.

3.3. Theorem. $\mathfrak{e} \geq \min \{\mathfrak{e}(\omega), \operatorname{cov}(\mathcal{M})\}$; thus either $\mathfrak{e}<\operatorname{cov}(\mathcal{M})$ or $\mathfrak{e}(\omega) \leq \operatorname{cov}(\mathcal{M})$ imply $\mathfrak{e}=\mathfrak{e}(\omega)$.

REMARK. The statement is very similar to a recent result of Kamburelis who proved $\mathfrak{s} \geq \min \{\mathfrak{s}(\omega), \operatorname{cov}(\mathcal{M})\}$, where $\mathfrak{s}$ is the splitting number and $\mathfrak{s}(\omega)$ the $\aleph_{0}$-splitting number.

Proof. The second statement easily follows from the first. To prove the latter, let $\mathcal{F} \subseteq \omega^{\omega},|\mathcal{F}|<\min \{\mathfrak{e}(\omega), \operatorname{cov}(\mathcal{M})\}$. We shall show $|\mathcal{F}|<\mathfrak{e}$. For $\sigma \in \omega^{<\omega} \backslash\{\langle\rangle\}$, we construct recursively sets $D^{\sigma} \subseteq \omega$ and predictors $\pi^{\sigma}=\left(D^{\sigma},\left\langle\pi_{n}^{\sigma} ; n \in D^{\sigma}\right\rangle\right)$ such that: 
(i) $D^{\sigma \nmid i} \supseteq D^{\sigma}$ for $i \in|\sigma|$;

(ii) for all $f \in \mathcal{F}$ and all $\sigma \in \omega^{<\omega}$ there is $i \in \omega$ so that $f$ is predicted by $\pi^{\sigma^{\wedge}\langle i\rangle}$.

First construct $\pi^{\langle i\rangle}=\left(D^{\langle i\rangle},\left\langle\pi_{n}^{\langle i\rangle} ; n \in D^{\langle i\rangle}\right\rangle\right)$ satisfying (ii) by applying $|\mathcal{F}|<\mathfrak{e}(\omega)$.

To do the recursion, assume $\pi^{\sigma}=\left(D^{\sigma},\left\langle\pi_{n}^{\sigma} ; n \in D^{\sigma}\right\rangle\right)$ is constructed for some fixed $\sigma \in \omega^{<\omega}$. Given $f \in \omega^{\omega}$, define $f^{\sigma}$ by:

$$
f^{\sigma}(i):=f\left(k_{i}^{\sigma}\right)
$$

where $\left\{k_{i}^{\sigma} ; i \in \omega\right\}$ is the increasing enumeration of the set $D^{\sigma}$. Let $\mathcal{F}^{\sigma}=\left\{f^{\sigma} ; f \in \mathcal{F}\right\}$. Again we get $\omega$ many predictors $\bar{\pi}^{\sigma^{\wedge}\langle i\rangle}=\left(\bar{D}^{\sigma^{\wedge}\langle i\rangle},\left\langle\bar{\pi}_{n}^{\sigma^{\wedge}\langle i\rangle} ; n \in \bar{D}^{\sigma^{\wedge}\langle i\rangle}\right\rangle\right), i \in \omega$, so that every $f^{\sigma} \in \mathcal{F}^{\sigma}$ is predicted by some $\bar{\pi}^{\sigma^{\wedge}\langle i\rangle}$. Let $D^{\sigma^{\wedge}\langle i\rangle}=\left\{k_{j}^{\sigma} ; j \in \bar{D}^{\sigma^{\wedge}\langle i\rangle}\right\}$. Fix $j \in \bar{D}^{\sigma^{\wedge}\langle i\rangle}$ and $s \in \omega^{k_{j}^{\sigma}}$. Let $\bar{s} \in \omega^{j}$ be defined by $\bar{s}(\ell)=s\left(k_{\ell}^{\sigma}\right)$. Put $\pi_{k_{j}^{\sigma}}^{\sigma^{\wedge}\langle i\rangle}(s):=\bar{\pi}_{j}^{\sigma^{\wedge}\langle i\rangle}(\bar{s})$. Now it is easy to see that $\pi^{\sigma^{\wedge}\langle i\rangle}$ predicts $f$ whenever $\bar{\pi}^{\sigma^{\wedge}\langle i\rangle}$ predicts $f^{\sigma}$. Thus (i) and (ii) hold. This completes the recursive construction.

Given $f \in \omega^{\omega}$, let $T_{f}=\left\{\sigma \in \omega^{<\omega} ;\right.$ for all $i \leq|\sigma|\left(\pi^{\sigma \nmid i}\right.$ does not predict $f$ everywhere)\}. By the above construction, the sets $\left[T_{f}\right]$ are nowhere dense for $f \in \mathcal{F}$. As $|\mathcal{F}|<\operatorname{cov}(\mathcal{M})$, there must be $g \in \omega^{\omega} \backslash \bigcup_{f \in \mathcal{F}}\left[T_{f}\right]$. Now use the Observation (3.2.) to construct a new predictor from the $\left\langle\pi^{g \uparrow n} ; n \in \omega\right\rangle$ which will predict all $f \in \mathcal{F}$.

3.4. It is unclear whether $\mathfrak{e}=\mathfrak{e}(\omega)$ can be proved in $Z F C$. In view of Theorem 3.3 it seems reasonable to ask first

Question. Is $\mathfrak{e}>\operatorname{cov}(\mathcal{M})$ consistent?

Of course, we may also consider the cardinal $\mathfrak{e}_{\ell}(\omega)$, the smallest size of a family $\mathcal{F}$ of functions from $\omega$ to $\omega$ such that no countable family of linear predictors predicts all $f \in \mathcal{F}$. However, it is now easy to see that $\mathfrak{e}_{\ell}(\omega)=\mathfrak{e}_{\ell}$. This is so because $\mathfrak{e}_{\ell}(\omega) \leq \min \{\mathfrak{e}(\omega), \mathfrak{b}\} \leq$ $\min \{\mathfrak{e}, \mathfrak{b}\} \leq \mathfrak{e}_{\ell}$. To see the first inequality, note that the argument for $\mathfrak{e}_{\ell} \leq \mathfrak{b}$ gives $\mathfrak{e}_{\ell}(\omega) \leq \mathfrak{b}$ as well (see [Br, section 5.4] for a stronger result); for the second inequality, $\min \{\mathfrak{e}(\omega), \mathfrak{b}\} \leq \operatorname{cov}(\mathcal{M})$ by rewriting Blass' $\min \{\mathfrak{e}, \mathfrak{b}\} \leq \operatorname{cov}(\mathcal{M})[\mathrm{Bl}$, Theorem 13] and thus $\min \{\mathfrak{e}(\omega), \mathfrak{b}\}=\min \{\mathfrak{e}(\omega), \operatorname{cov}(\mathcal{M}), \mathfrak{b}\} \leq \min \{\mathfrak{e}, \mathfrak{b}\}$ by Theorem 3.3; the third inequality is Lemma 2.2.

3.5. Duality. Most of the cardinal invariants of the continuum come in pairs and results about them usually can be dualized (see [Br, section 4.5] for details). In our situation, the dual cardinals are: the dominating number $\mathfrak{d}$ (dual to $\mathfrak{b}$ ), the smallest size of a family 
$\mathcal{F} \subseteq \omega^{\omega}$ such that given any $g \in \omega^{\omega}$ there is $f \in \mathcal{F}$ with $g \leq^{*} f$; the (linear) covering number $\operatorname{cov}(\mathcal{J})\left(\operatorname{cov}\left(\mathcal{J}_{\ell}\right)\right)$ of the ideal $\mathcal{J}\left(\mathcal{J}_{\ell}\right)$ (the first being dual to both $\mathfrak{e}$ and $\mathfrak{e}(\omega)$, the second being dual to $\mathfrak{e}_{\ell}$ ), the least cardinality of a family of (linear) predictors $\Pi$ such that every function $f \in \omega^{\omega}\left(\mathbb{Z}^{\omega}\right)$ is predicted by some $\pi \in \Pi$. Then we get:

TheOrem. (a) It is consistent with $Z F C$ to assume $\mathfrak{d}>\operatorname{cov}(\mathcal{J})$.

(b) $\operatorname{cov}\left(\mathcal{J}_{\ell}\right)=\max \{\operatorname{cov}(\mathcal{J}), \mathfrak{d}\}=\min \left\{|\mathcal{S}| ; \mathcal{S}\right.$ consists of slaloms $S_{D}^{\bar{a}}$ where $\bar{a}=\left\langle a_{n} \in\right.$ $[\omega] \leq n ; n \in D\rangle$ and $D \subseteq \omega$ is infinite and $\left.\forall f \in \omega^{\omega} \exists S_{D}^{\bar{a}} \in \mathcal{S} \forall^{\infty} n \in D\left(f(n) \in a_{n}\right)\right\}$.

PROOF. These dualizations are standard, and we therefore refrain from giving detailed proofs. The model for (a) is gotten by iterating the p.o. $\mathbb{P}$ from $\S 1 \omega_{1}$ times with finite support over a model for $M A+\neg C H$. (b) is the dual version of Theorem $\mathrm{B}$ and Lemma 2.5 .

We close our work with a diagram showing the relations between the cardinal invariants considered in this work (in particular, the Specker-Eda number $\mathfrak{s e}$ and the evasion number $\mathfrak{e}$ ) and some other cardinal invariants of the continuum (in particular, those of Cichon's diagram). We refer the reader to $[\mathrm{Bl}],[\mathrm{Br}]$ or $[\mathrm{Fr}]$ for the cardinals not defined here. A similar diagram was drawn in [Br, section 4].

$\operatorname{cov}(\mathcal{L}) \quad \operatorname{unif}(\mathcal{M}) \quad \operatorname{cof}(\mathcal{M}) \quad \operatorname{cov}\left(\mathcal{J}_{\ell}\right) \quad \operatorname{cof}(\mathcal{L})$

$\mathfrak{d} \operatorname{cov}(\mathcal{J})$

$\mathfrak{e} \quad \mathfrak{b}$

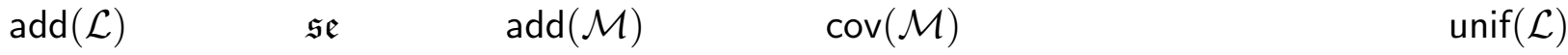

$\mathfrak{p}$

In the diagram, cardinals increase as one moves up and to the right. To enhance readability, we omitted the relations $\mathfrak{e} \leq \operatorname{unif}(\mathcal{L})$, and its dual $\operatorname{cov}(\mathcal{L}) \leq \operatorname{cov}(\mathcal{J})$. The dotted lines give the relations $\operatorname{add}(\mathcal{M})=\min \{\mathfrak{b}, \operatorname{cov}(\mathcal{M})\}, \mathfrak{s e}=\min \{\mathfrak{e}, \mathfrak{b}\}$, and their dual versions. 


\section{References}

[Ba 1] T. BArtoszyński, Additivity of measure implies additivity of category, Transactions of the American Mathematical Society, vol. 281 (1984), pp. 209-213.

[Ba 2] T. BARtoszyński, Combinatorial aspects of measure and category, Fundamenta Mathematicae, vol. 127 (1987), pp. 225-239.

[Bl] A. Blass, Cardinal characteristics and the product of countably many infinite cyclic groups, Journal of Algebra (to appear).

[Br] J. BREndLE, Evasion and prediction - the Specker phenomenon and Gross spaces, Forum Mathematicum (to appear).

[Fr] D. Fremlin, Cichoń's diagram, Séminaire Initiation à l'Analyse (G. Choquet, M. Rogalski, J. Saint Raymond), Publications Mathématiques de l'Université Pierre et Marie Curie, Paris, 1984, pp. 5-01 - 5-13.

[JS] H. Judah And S. Shelah, The Kunen-Miller chart (Lebesgue measure, the Baire property, Laver reals and preservation theorems for forcing), Journal of Symbolic Logic, vol. 55 (1990), pp. 909-927.

[Mi] A. Miller, Some properties of measure and category, Transactions of the American Mathematical Society, vol. 266 (1981), pp. 93-114.

[Sp] E. SpeckeR, Additive Gruppen von Folgen ganzer Zahlen, Portugaliae Mathematica, vol. 9 (1950), pp. 131-140. 